\title{
A Composite Detection and Disposal Scheme for Explosive Embedded in Building
}

\author{
Xiang Fang \\ Engineering Institute of Engineering Corps, PLA University of Science and Technology \\ 1 Hai Fu Xiang Street, Nanjing 210007, China \\ Tel: 86-25-8082-1444Ｅ-mail: nanjingfangxiang@163.com \\ Dong Li (Corresponding author) \\ Engineering Institute of Engineering Corps, PLA University of Science and Technology \\ 1 Hai Fu Xiang Street, Nanjing 210007, China \\ Tel: 86-137-7669-1036 E-mail: nanjinglidong@sohu.com \\ Lijun Xie \\ Engineering Institute of Engineering Corps, PLA University of Science and Technology \\ 1 Hai Fu Xiang Street, Nanjing 21007, China \\ Tel: 86-137-7668-3213Ｅ-mail: 381596054@qq.com
}

Received: March 12, 2011

Accepted: April 20, 2011

doi:10.5539/mas.v5n3p136

\begin{abstract}
The terrorists may embed explosives in the building, and then detonate them at a certain time. In recent years, the detection of this kind of explosive has been a new subject in anti-terrorism. Aiming to prevent this form of terrorist attack, this paper discusses some current explosive detection techniques and the way they may be used in the inspection and assessment of building. The techniques that have been studied are: ground penetration radar, harmonic radar, neutral technique and abrasive water jet cutting technology. Based on the results of analysis----an overall assessment scheme is proposed. This type of scheme has been proved to fit environment of reinforced concrete and to improve detection rate as well as for reduction of false rates too.
\end{abstract}

Keywords: Explosive detection and disposal, Ground penetration radar, Harmonic radar, Neutral technology, Abrasive water jet cutting, Anti-terrorism

\section{Introduction}

Explosion activities are the main forms of terrorism in the world today. Its existence and spread influenced security and stability of people and communities worldwide. The terrorists may make the explosives into preformed units in advance, or embed them in the reinforced concrete, or in the hollow structure of buildings (e.g. mezzanines), then detonate them at certain time. Detection techniques are now available in airport security, battlefield mine detection, industry nondestructive testing etc. i.e. that are applicable to building structure. Until so far, the research community has mainly concentrated on the development of specific detection technique. In consideration of new security situation, a more integrated detection-disposal method and approach is needed. The paper analyzes three types of detection techniques and one disposal explosive technique applicable to detect explosives embedded in the buildings. Based on the analysis results, a composite scheme is proposed. This scheme has been successfully applied to assess stadiums in the 2008 Beijing Olympic Games and the 2010 Shanghai World EXPO.

\section{Detection circumstances and Characteristics of explosives}

\subsection{Detection circumstances}

Reinforced concrete is the key material of modern architecture. The outer walls are usually built with hollow bricks, concrete blocks or reinforced concrete (RC). Floors, passageways and stairs are usually also built with concrete, blocks or RC. The thickness of a RC wall is usually between 37 up to 49 centimeters. The load-bearing 
column and load-bearing beam are usually square or cylindrical. The length of the loading-bearing beams is usually 40 to 60 centimeters, or sometimes up to 80 centimeters. The diameter of the support structure can reach beyond 1.5 meters. The thickness of concrete foundation do not exceed 1 meter normally.

\subsection{Characteristics of explosives embedded in buildings}

Explosives are normally composed of dynamite, a detonating device, a metal or nonmetallic case. Terrorists may embed them ahead in selected strategic locations, such as important buildings and site (e.g. official platforms, key gateways, bearings wall or beams etc.). In this way, they could decide for a certain time and opportunity to detonate the explosives. With regard to buildings, the characteristics of the explosives are as the following:

(1)The explosives have a regular shape and quality, which are different from its surroundings. For example, the density of dynamite is $1.4-2.0 \mathrm{~g} / \mathrm{cm}^{3}$ and of concrete $2200-2400 \mathrm{~kg} / \mathrm{m}^{3}$.

(2)Dynamite is rich in element $\mathrm{N}$, but generally the construction materials do not contain element $\mathrm{N}$. It can be identified as an important basis of explosives characteristics.

(3)The Detonating device contains a PN node and metal-oxide-metal, but the architectural itself do not contain semiconductor devices. This is an important basis for judging timing or remote detonating device. Because they contain a number of $\mathrm{PN}$ nodes.

(4)There are great differences between the dielectric constant of explosives and the concrete medium. These features can be used in ground penetration radar imaging to identify explosives and their position.

\section{Analysis of detection and disposal techniques}

\subsection{Shallow ground penetrating radar}

Ground Penetrating Radar (GPR) is a new kind of non-destructive detecting technique, which can detect underground and buried structure. Unlike common radar reconnaissance techniques, GPR detect targets by the media. It launches a form of electromagnetic waves through the ground, which are reflected underground at the interfaces of the dielectric properties, which are changed, and then return to ground. In the process of transmission, the intensity of the electromagnetic field and the form of the electromagnetic wave will vary with the change of dielectric properties and geometric shapes. According to the echo signal delay, shape, spectral characteristic and other parameters, it is possible to interpret the underground target depth, the structure's medium and its properties. A simplified schematic diagram is shown in Figure.1. Penetration characteristics of electromagnetic waves in a variety of media were made by HAETC (Hughes Advance Electromagnetic Technology Center) (Kowalsky, 2001, p.1615). At this time, a diagram showing the relationship between attenuation and frequency of electromagnetic wave propagation in a variety of media was drawn (Figure 2). It shows that the attenuate of electromagnetic waves is the most characteristic through concrete (Abid Ali Shah, 2007, p.1-5). Wave form and grayscale image of GRP can be seen in Figure.3 and Figure.4

\subsection{Principles of harmonic radar}

The harmonic radar technique is used to detect a harmonic of nonlinear goal. Typical nonlinear goals include the electronic equipment with PN nodes and the objects with metal junctions. Voltage current characteristic of nonlinear targets is curved line relation. For PN nodes of semiconductor devices, its Voltage current characteristic is shown in Figure.5. When two kinds of metal contact, the metal nodes or metal-oxide-metal nodes can be produced under proper condition (such as pressure, distance), especially if there is a layer of oxide (e.g. rust) in the middle (Junzhe Yang, 2005, p.1235). Its Voltage current characteristic can observed directly using transistor, as shown in Figure 6.

\subsection{Neutron detection technology}

At present, neutron detection techniques that are used to detect hidden explosives are based on X-ray, chemical vapor, nuclear quadrupole resonance and neutron techniques. Among them, X-ray projection imaging technique can be used to identify density resolution of goods, but it is unable to identify element component of the hidden substances. Although chemical vapor analysis has high sensitivity, it is difficult to detect dynamite that has a low vapor pressure. Nuclear quadrupole resonance technique can be used to classify dynamite, but it is easily effected by external electromagnetic waves and may give invalid results in terms of liquid explosives detection (Andy Buffler, 2004, p.853).

Neutron detection is a type of energy explosives detection technique. It launches a number of Neutron beams to the object for inspection. It can penetrate concrete and it is not charged. It is harmless to any object and material which are irradiated. But neutron beams are extremely sensitive to Nitrogen atoms, which are particular high in dynamite. When suspicious objects are irradiated by neutron beams, they will give off gamma ray immediately. 
Base on this statement, it is possible to detect the existence of explosives scientifically and within a short time (F. L. Chen, 2002, p.717). The most common dynamite chemicals and physical properties are shown by table 1 .

\subsection{Abrasive water jet cutting technique}

Abrasive water jet cutting technology is a special and rapidly developing methodology. Because of its particular processing characteristics: its high efficiency processing, wide adaptability and sensitivity with regard to environment protection, it has unique advantages in cutting heat sensitive materials and hard brittle materials (F. L. Chen, 2001, p.1479).

An abrasive water jet cutting system is composed of several subsystems, including power system, water supply system, hydraulic system, abrasive supply system and cutting device, etc. Firstly, a high-pressure pump transforms the water out of tank into high-pressure water by use of engine power. Secondly, high-pressure water goes through hose to mix abrasive coming from the abrasive can, then flow through the nozzle of the system. At last, the accelerated mixed liquid squirt and operate cutting implement (LIU H, 2004, p.488). This process above is shown in Figure 7: 1.water tank 2.filter 3.piston pump 4.pressure gauge 5.relief valve 6.throttle 7.one-way valve 8 .mixing-cavity 9 .globe valve 10 .abrasive can 11 .nozzle.

\subsection{Analysis of detection techniques}

Based on the above, each detecting technology has its advantages and limitations.

(1) The equipment of GPR is simple and easy to use. GPR can detect explosives in different depths using different frequencies. The detection will only be a failure when depth of the detection is out of range. Water content of the target will reduce detection depth and detection effect.

(2) Due to high sensitivity of detection to non-linear node, harmonic radar has the advantage to detect remote control or timing detonating device. Its limitation is that it is insensitive to other kinds of explosive and can shield detonating device.

(3) Neutron technology is also one of the most important techniques, because it can be used to judge the existence of the explosive by its special physical properties for element $\mathrm{N}$.

\section{A composite detection and disposal scheme}

\subsection{How to improve the detection effect}

Based on the discussion and analysis above, it is difficult to detect the existence of explosives by any single way method to the limitations of each detection technology.

(1) According to the characteristics of all kinds of detection technologies, composite systems must be established and complementarities may be formed. Composite system can play integral advantages to reduce detecting false alarm rate and to improve detection rate.

(2) According to the characteristics and circumstances of detection targets, strong pointed system can be established.

(3) There is a need to improve the knowledge and skill of operating personnel. Since there are differences among detection technology, there is a need to know how to eliminate the false and based on t experience and professional qualities. Therefore continuous training in this area is needed.

\subsection{Establish of a composite detection and disposal mechanism for suspicious explosives}

Explosives have many characteristics, which can be detected in built structures and environments. GPR technique can detect foreign characteristics due to electromagnetic differences between explosives and the environment of building structure. The harmonic radar technique can detect detonating devices and give an alarm. Neutron technique can further confirm the existence of explosives. Based on the assumptions above, a composite detection system is formed and an enhanced explosive detecting process can be established. This process includes four stages, as follows:

(1) Rough detecting: GPR and harmonic radar can be used to assess building the structure roughly and quickly. If suspicious targets are found, we can move on to the second stage.

(2) Fine detecting: GPR detects suspicious target with fine mode, and produces sectional and layouts image with the use of a computer. When analyzing the image, information regarding the depth and size of suspicious can be obtained.

(3) Confirmation: the neutron detection can detect if there area any $\mathrm{N}$ elements embedded in the concrete. If the above conditions are met, the presence of explosive may be confirmed. 
(4) Disposal: abrasive water jet cutting system may be used to disposal explosives embedded in the buildings.

\section{Summary}

In conclusion, facing with such a special environment, it is hard to achieve totally accurate and reliable results just by a single detection method. Rather, a comprehensive method is need. The detection and disposal of explosives is a difficult, dangerous and complex process. It's an arduous task and an issue of large properties, especially considering the security situation in the world. Therefore, trained explosive-handling personnel with proper experience is needed; i.e. that can master these basic technologies, process and skills in practice, and that can deal with changing situations.

\section{References}

Abid Ali Shah and Yuri Ribakow. (2007). Non-destructive measurements of crack assessment and defect detection in concrete structures, Materials and Design, 2007, 22(5):1-9.

Andy Buffler. (2004). Contrband detection with fast neutrons. Radiation Physics and Chemistry, 2004(71):853-861.

F. L. Chen and E. Siores. (2001). The effect of cutting jet wariation on striation formation in abrasive water jet cutting. International Journal of Machine Tools and Manufacture, 2001(41):1479-1486.

F. L. Chen, E.Siores and K. Patel. (2002). Improving the cut surface qualities using different controlled nozzle oscillation techniques. International Journal of Machine Tools and Manufacture, 2002(42):717-722

Junzhe Yang, Chishan Yu and Chihwen Liu. (2005). A new method for power signal harmonic analysis. IEEE Transactions on Power Delivery, 2005(2):1235-1239.

Kowalsky M B, Dietrich P, Teutsch G and Rubin Y. (2001). Forward modeling of ground-penetrating radar data using digitized outcrop images and multiple scenarios of water saturation. Water Resources Research, 2001, 37(6):1615-1625.

LIU H, WANGA J and KELSONB N, et al. (2004). A study of abrasive water jet characteristics by CFD simulation. Journal of Materials Processing Technology, 2004(153-154):488-493.

Table 1. Common dynamite chemical and physical properties

\begin{tabular}{|c|c|c|c|c|}
\hline Dynamite & Molecular formula & $\begin{array}{c}\text { Molecular } \\
\text { weight }\end{array}$ & $\begin{array}{c}\text { N element content } \\
(\%)\end{array}$ & $\begin{array}{c}\text { Density } \\
(\mathrm{g} / \mathrm{cm})\end{array}$ \\
\hline TNT & $\mathrm{C}_{6} \mathrm{H}_{2}\left(\mathrm{NO}_{2}\right) \mathrm{CH}_{3}$ & 227 & 18.5 & 1.65 \\
\hline AN & $\mathrm{NH}_{4} \mathrm{NO}_{3}$ & 80 & 35.0 & 1.59 \\
\hline RDX & $\left(\mathrm{CH}_{2} \mathrm{NNO}_{2}\right)_{3}$ & 222 & 38.0 & 1.83 \\
\hline HMX & $\left(\mathrm{CH}_{2} \mathrm{NNO}_{2}\right)_{4}$ & 296 & 38.0 & 1.96 \\
\hline PETN & $\mathrm{C}_{5} \mathrm{H}_{8}\left(\mathrm{ONO}_{2}\right)_{4}$ & 316 & 17.7 & 1.78 \\
\hline TE & $\mathrm{C}_{6} \mathrm{H}_{2}\left(\mathrm{NO}_{2}\right)_{4} \mathrm{NCH}_{3}$ & 287 & 24.4 & 1.73 \\
\hline
\end{tabular}

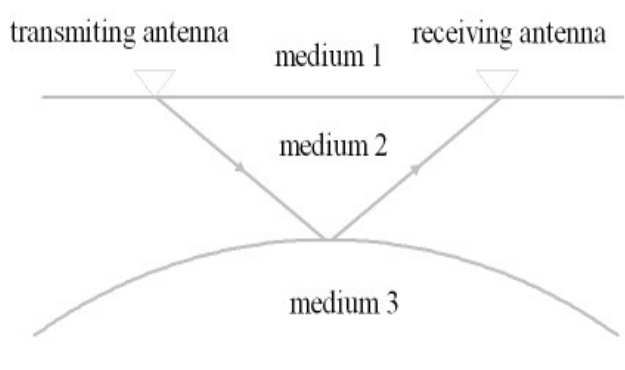

Figure 1. Schematic Diagram of GPR

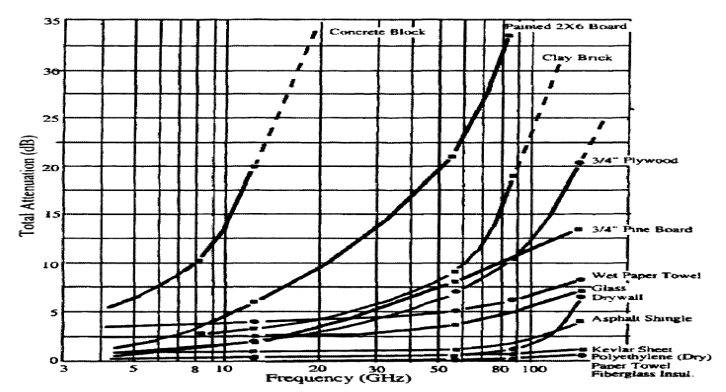

Figure 2. Attenuate of Electromagnetic Wave 


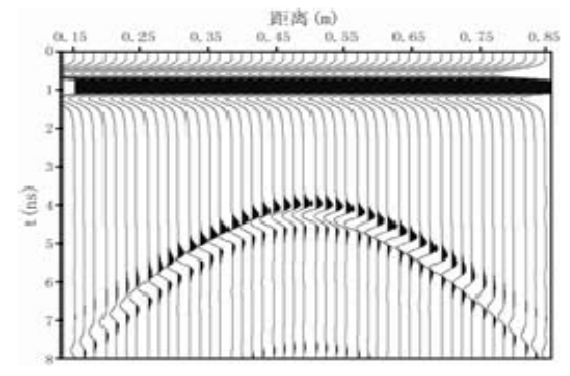

Figure 3. Wave Form of GPR

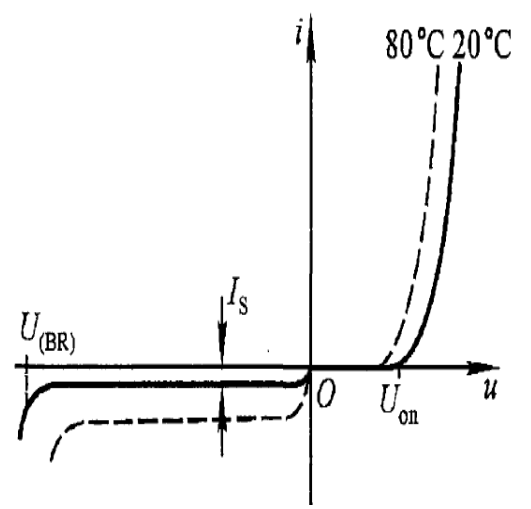

Figure 5. Voltage Current Characteristic of PN Node

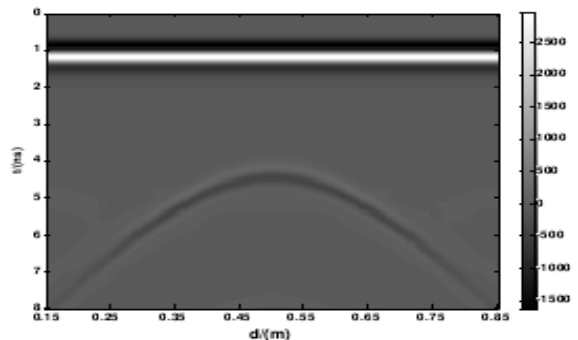

Figure 4. Grayscale Image of GPR

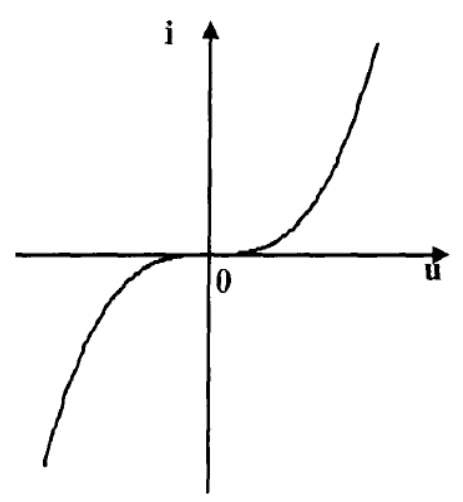

Figure 6. Voltage Current Characteristic of Metal Nodes

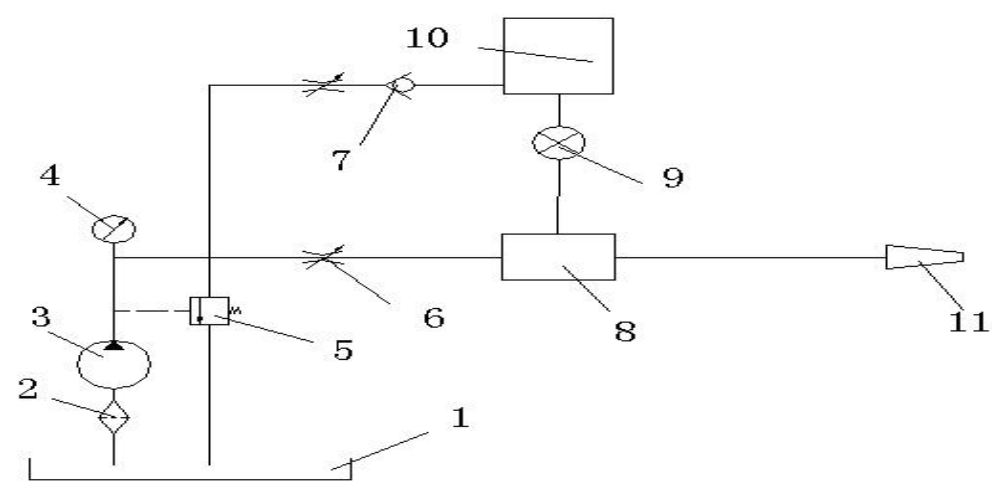

Figure 7. Flow Chart of Abrasive Water Cutting System 